DOI: http://dx.doi.org/10.12957/demetra.2014.9485

\title{
A segurança alimentar e nutricional do público infanto-juvenil: o leite como componente
}

\section{Food security of the juvenile population: milk as a component}

\author{
Thais Regina Mezzomo' \\ Juliana Nadal' \\ 1 Programa de Pós-graduação em Segurança \\ Alimentar e Nutricional. Universidade Federal do \\ Paraná. Curitiba, PR, Brasil. \\ Correspondência/Correspondence \\ Thais Regina Mezzomo \\ E-mail: thaismezzomo@yahoo.com.br
}

\section{Resumo}

O leite materno é o primeiro alimento do recém-nascido, o único capaz de dobrar o peso do lactente nos primeiros meses de vida e promover forte desenvolvimento cerebral. Entretanto, outros leites, como o bovino, costumam ser inseridos na alimentação infantil. Assim, sabendo que o direito de crescer com a melhor saúde possível é um direito fundamental de toda criança e está contemplado na Convenção dos Direitos da Criança, se faz necessário conhecer a importância do leite bovino na segurança alimentar e nutricional de crianças e adolescentes. Para tanto, foi realizado levantamento bibliográfico nas bases de dados SciELO e MEDLINE referentes à última década. Nesta revisão, foi possível observar que os produtos lácteos contribuem com aproximadamente $50 \%$ das necessidades diárias de cálcio no mundo todo e contribuem principalmente para o desenvolvimento da saúde óssea e crescimento saudável das crianças maiores de dois anos de idade. No entanto, a literatura demonstra relação entre consumo de leite bovino e ocorrência de anemia, de alergias alimentares e de diabetes tipo 1. Assim, observa-se que as práticas de alimentação são determinantes das condições de saúde na infância e estão condicionadas ao poder aquisitivo das famílias. Desta forma, políticas públicas em todas as regiões do país devem ser implantadas a fim de assegurar que todas as crianças e adolescentes brasileiros sejam supridos com quantidade e qualidade necessárias não apenas desse alimento, mas de todos os outros, de acordo com sua faixa etária, garantindo assim, a Segurança Alimentar e Nutricional do público infanto-juvenil.

Palavras-chave: Crianças. Leite. Desenvolvimento Infantil. Segurança Alimentar e Nutricional. 


\section{Abstract}

Breast milk is the first food of the newborn, the only one capable of doubling the weight of the infant in its first months of life and carrying out strong brain development. However, other kinds of milk, such as bovine milk, are usually embedded in infant feeding. Thus, it has been known that the right to grow up with the best possible health is a fundamental right of every child and provided by the Convention on the Rights of the Child, it is necessary to know the importance of bovine milk in the food security of children and adolescents. Therefore, a literature survey was conducted in SciELO and MEDLINE data for the last decade. In this review it was observed that dairy products provide about $50 \%$ of daily calcium needs worldwide and mainly contribute to the development of healthy bones and healthy growth of children over 2 years old. However, the literature shows the relationship between bovine milk consumption and the occurrence of anemia, food allergies and type 1 diabetes. Thereby, it is observed that feeding practices are determinants of health in childhood and they are subject to the purchasing power of households. So public policies in all regions of the country should be deployed to ensure that all Brazilian children and adolescents are supplied with necessary quantity and quality not only about milk, but all other kinds of food, according to their age, thus ensuring Food Security of the juvenile population.

Key words: Child. Milk. Child Development. Food Security.

\section{Introdução}

A nutrição adequada é um dos fatores de maior impacto na saúde infantil, principalmente pela influência decisiva que o estado nutricional exerce sobre os riscos de morbimortalidade, o crescimento e o desenvolvimento. ${ }^{1}$ Segundo Best et al., ${ }^{2}$ a prevalência de desnutrição em países da América Latina é menor que 10\%. No Brasil, dados da Pesquisa de Orçamentos Familiares (POF) mostram que o déficit de peso em 2008-2009 em crianças menores de cinco anos é de 6\%, com ênfase no primeiro ano de vida $(8,9 \%)$. Nas crianças de cinco a nove anos, o déficit de peso acomete 4,1\%, e o déficit de altura atinge 6,8\% desta população. Em adolescentes, entre 10 e 19 anos, o déficit de peso é de 3,35\%. ${ }^{3}$ Além disso, a prevalência de crianças com anemia e deficiência de vitamina A é de $20,9 \%$ e $17,4 \%$, respectivamente. ${ }^{4}$ 
Nesse contexto, surge a Segurança Alimentar e Nutricional (SAN) como uma garantia de acesso contínuo à quantidade e qualidade suficientes de alimentos obtidos por meio socialmente aceitável, de forma a assegurar o bem-estar e a saúde dos indivíduos. ${ }^{1} \mathrm{O}$ conceito de SAN deve abranger além do acesso aos alimentos, conservação da biodiversidade, promoção da saúde e da nutrição, qualidade sanitária e biológica dos alimentos e promoção de práticas alimentares saudáveis - a produção de conhecimento e o acesso à informação. ${ }^{5}$ Assim, a SAN vem sendo avaliada e utilizada em estudos populacionais, os quais ocupam de forma crescente a agenda pública brasileira, devido, entre outros, ao fato de que a alimentação adequada é um direito fundamental do ser humano e indispensável à realização dos direitos consagrados pela Constituição Federal. ${ }^{6}$

Recentemente, a Pesquisa Nacional por Amostra de Domicílios (PNAD) avaliou a insegurança alimentar na população brasileira e encontrou que crianças e adolescentes (até 17 anos) apresentam $42,75 \%$ de insegurança alimentar, sendo 7,9\% consideradas em insegurança alimentar grave, segundo a classificação da Escala Brasileira de Insegurança Alimentar. ${ }^{5}$

O leite materno é o primeiro alimento do recém-nascido, o único capaz de dobrar o peso do lactente nos primeiros seis meses de vida e de promover forte desenvolvimento cerebral. ${ }^{7}$ Entretanto, a partir dos dois anos de idade, outros leites, como o bovino, costumam ser inseridos na alimentação infantil por serem fontes alimentares concentradas em macro e micronutrientes, ${ }^{8}$ fazendo do ser humano o único mamífero que utiliza leite de outras espécies para sua nutrição.

Se, por um lado, estudos demonstram que esse consumo já existe desde a Idade do Cobre, há aproximadamente seis mil anos, ${ }^{7}$ por outro, sabe-se que o leite bovino pode levar ao desenvolvimento de anemia e alergias alimentares nesta população. Assim, sabendo que o direito de crescer com a melhor saúde possível é um direito fundamental de toda criança e está contemplado na Convenção dos Direitos da Criança, ${ }^{9}$ se faz necessário conhecer a importância do leite bovino na segurança alimentar e nutricional de crianças e adolescentes.

\section{Método}

Foi realizada pesquisa bibliográfica na base de dados Scientific Eletronic Library On-line (SciELO) e Medical Literature Analysis and Retrieval System Online (MEDLINE), utilizando a associação entre os descritores "leite", "segurança alimentar e nutricional" e "crianças", em português e em inglês, referentes à última década. Os descritores "leite" e "políticas públicas" foram pesquisados apenas na SciELO, devido à abrangência de periódicos científicos brasileiros. 
Foram encontrados 86 artigos contemplando os referidos descritores. Os critérios de exclusão empregados foram: leite não bovino, público adulto e políticas públicas que não envolviam diretamente o leite bovino. Desta forma, foram inseridos no presente trabalho 23 artigos. Além disso, foram incluídos dois livros, um artigo publicado antes do ano de 2002, uma tese, duas diretrizes de recomendação nutricional, quatro legislações vigentes e cinco pesquisas de âmbito nacional, consideradas relevantes sobre o assunto, foram citados na revisão.

\section{Recomendações de macronutrientes e cálcio para crianças e adolescentes}

Para o crescimento e o desenvolvimento infantil adequado, a alimentação deve ter um balanço entre a distribuição dos macronutrientes. As fontes de energia devem ser 55 a $60 \%$ provindas de carboidratos, e 25 a 30\% de lipídios. Os requerimentos proteicos por quilograma de peso na criança são maiores em relação aos adultos, necessitando ainda de maior proporção de aminoácidos essenciais. Os valores variam com a faixa etária e o sexo entre $0,85 \mathrm{~g} / \mathrm{kg} / \mathrm{dia}$ a $1,52 \mathrm{~g} / \mathrm{kg} / \mathrm{dia} .^{10}$

A recomendação de cálcio nos primeiros seis meses é de $210 \mathrm{mg}$, e dos sete aos 12 meses, de $270 \mathrm{mg} .{ }^{11}$ Esses valores se baseiam na quantidade contida no leite materno, o qual representa a principal fonte alimentar no primeiro ano de vida. ${ }^{12}$ Já as recomendações diárias (RDA) de cálcio para as faixas etárias de 1-3 anos e 4-8 anos são de 700 e $1.000 \mathrm{mg}$, respectivamente, ${ }^{11}$ facilmente obtidas com a ingestão de três a quatro porções de produtos lácteos por dia. Em adolescentes, segundo recomendações do Food and Nutrition Board of the Institute of Medicine (1997-2001), a RDA de cálcio é de $1.300 \mathrm{mg}$, distribuídas em quatro porções diárias do grupo de lácteos. ${ }^{11}$

Os produtos lácteos contribuem com $47 \%$ da ingestão de cálcio na população americana. ${ }^{13}$ Já na Holanda, $73 \%$ do consumo de cálcio das crianças advêm de produtos lácteos. ${ }^{14}$ Outro fator igualmente importante, relativo à quantidade de cálcio presente na porção alimentar, é sua biodisponibilidade. O quadro 1 apresenta as fontes alimentares de cálcio de acordo com sua absorção. ${ }^{12}$ 
Quadro 1. Fontes alimentares de cálcio de acordo com sua absorção

\begin{tabular}{|c|c|c|c|c|c|}
\hline $\begin{array}{c}\text { Fonte } \\
\text { Alimentar }\end{array}$ & Porção (g) & $\begin{array}{c}\text { Conteúdo } \\
\text { de Cálcio } \\
(\mathrm{mg} / \text { porção })\end{array}$ & $\begin{array}{c}\text { Absorção } \\
\text { estimada }(\%)\end{array}$ & $\begin{array}{c}\text { Cálcio } \\
\text { absorvido } \\
\text { por porção } \\
(\mathrm{mg})\end{array}$ & $\begin{array}{c}\text { Porções } \\
\text { equivalentes } \\
\text { a 1 copo de } \\
\text { leite }\end{array}$ \\
\hline Leite & 240 & 290 & 32,1 & 93 & 1,0 \\
\hline Iogurte & 240 & 300 & 32,1 & 96,3 & 1 \\
\hline $\begin{array}{c}\text { Queijo } \\
\text { cheddar }\end{array}$ & 42 & 303 & 32,1 & 97,2 & 1 \\
\hline $\begin{array}{c}\text { Feijão } \\
\text { branco }\end{array}$ & 110 & 113 & 21,8 & 24,7 & 3,9 \\
\hline $\begin{array}{c}\text { Feijão } \\
\text { vermelho }\end{array}$ & 172 & 40,5 & 24,4 & 9,9 & 9,7 \\
\hline Brócolis & 71 & 35 & 61,3 & 21,5 & 4,5 \\
\hline Couve & 85 & 61 & 49,3 & 30,1 & 3,2 \\
\hline Espinafre & 85 & 115 & 5,1 & 5,9 & 16,3 \\
\hline $\begin{array}{c}\text { Pão } \\
\text { integral }\end{array}$ & 28 & 20 & 82 & 16,6 & 5,8 \\
\hline
\end{tabular}

Fonte: Adaptado de Silva; Mura (2010).

\section{Leite e seus efeitos na saúde}

Embora a exata composição varie em resposta a fatores como nutrição do animal e estágio de lactação, o leite bovino é composto por aproximadamente $87 \%$ de água, $5 \%$ de lactose, $3 \%$ de proteína, $4 \%$ de lipídeos e $0,7 \%$ de sais minerais..$^{15}$ Dentre os minerais, o leite contém elevada quantidade de fósforo, potássio, magnésio e cálcio, e pequena quantidade de vitamina $\mathrm{D}^{7}{ }^{716}$ Poucos alimentos contêm naturalmente a vitamina D, que desempenha papel no crescimento ósseo, juntamente com o cálcio, ${ }^{17-20}$ além de ser de interesse na prevenção do raquitismo, da osteomalácea, ${ }^{21}$ cardiomiopatias e, possivelmente, na prevenção de diabetes, hipertensão, dislipidemia, asma, ${ }^{22}$ doenças infecciosas e até mesmo alguns tipos de câncer. ${ }^{23}$ A deficiência dessa vitamina está se tornando uma epidemia mundial. ${ }^{22}$ 
O cálcio é o mineral mais abundante no corpo humano, sendo o nutriente responsável pela formação, manutenção e mineralização. ${ }^{24}$

A alta biodisponibilidade de cálcio dos produtos lácteos está relacionada com o conteúdo de vitamina D e a presença de lactose, as quais aumentam a absorção intestinal de cálcio. ${ }^{25}$ Estudos recentes mostram que a elevada biodisponibilidade desse elemento também se deve aos peptídeos bioativos que são liberados durante a digestão gastrointestinal do leite, principalmente os caseinofosfopeptídeos, os quais apresentam sítios de ligação com minerais divalentes $(\mathrm{Ca}, \mathrm{Mg}$ e Fe) e oligoelementos ( $\mathrm{Zn}, \mathrm{Ba}, \mathrm{Cr}, \mathrm{Ni}, \mathrm{Co}$ e Se). ${ }^{26}$ Além disso, os peptídeos bioativos conferem atividades antimicrobiana, antitrombótica, imunomoduladora, citomodulatória, influenciam a pressão arterial, o humor e também modulam a microbiota intestinal, desempenhando papelchave na defesa contra infecções e desenvolvimento de alergias em crianças alimentadas com fórmulas infantis. ${ }^{15}$

O leite bovino apresenta inúmeros benefícios à saúde. Vilela ${ }^{27}$ refere que o leite aumenta a absorção de retinol e cálcio, modula a resposta imunológica, aumenta a função antioxidante e antimicrobiana. E ainda, durante a digestão gastrintestinal, peptídeos são liberados das proteínas lácteas e apresentam efeitos regulatórios sobre várias funções corporais, tais como ação antihipertensiva, antioxidante, antitrombótica, hipocolesterolemiante, absorção de minerais, diminuição do apetite, aumento do trânsito intestinal com atividade antidiarreica, imunomodulatória, citomodulatória e antimicrobiana. ${ }^{15,26}$ É também fonte de energia e indispensável à síntese de esfingolipídios, essenciais para o desenvolvimento e funcionamento dos neurônios cerebrais. ${ }^{7}$

Por outro lado, tem sido demonstrado que o consumo de leite bovino apresenta-se como um consistente fator de risco para a ocorrência de anemia em crianças, pois pode interferir na absorção de ferro proveniente de outros alimentos. Vários estudos apontam que a ocorrência de anemia associada ao consumo de leite bovino tem como causas o aleitamento materno não exclusivo até o sexto mês de vida do lactente, a introdução de alimentos complementares não realizada conforme preconizado pelo Ministério da Saúde e, ainda, o uso de leite bovino como única fonte alimentar para o lactente, devido a ausência do leite materno e falta de acesso ou de informação sobre as fórmulas lácteas. ${ }^{28-30}$

Desta forma, se faz necessário manter programas de orientação à população sobre a importância do aleitamento materno exclusivo até os seis meses de vida do lactente e sobre a introdução dos alimentos complementares. Ainda, monitorar o consumo diário de leite para que o mesmo não ultrapasse as porções diárias recomendadas também é necessário; do mesmo modo, o leite não deve substituir as principais refeições (almoço e jantar) do dia. ${ }^{31}$ Corroborando esses dados, o Institute of Medicine reforçou que níveis superiores de cálcio não demonstraram benefícios maiores, e de fato, eles têm sido associados a outros problemas de saúde. ${ }^{11}$ 
Outra questão relacionada ao leite bovino é a ocorrência de alergias alimentares. ${ }^{32} \mathrm{O}$ uso abusivo do leite bovino como substituto do leite humano levou ao aumento da incidência de sensibilidade aos componentes desse leite. ${ }^{33}$ Sabe-se que a exposição precoce é associada ao aumento do risco de desenvolver diabetes melitus tipo 1 e doenças atópicas como a asma e outros tipos de alergias, cujos efeitos parecem persistir até a primeira década de vida da criança. ${ }^{28}$ Atualmente, cerca de $20 \%$ das alergias alimentares parecem ser consequência da alimentação com leite bovino, ${ }^{28}$ sendo que esta incidência está situada entre 1,9 e 7,5\%. ${ }^{33}$ Deste modo, o leite bovino não é recomendado antes do primeiro ano de vida. ${ }^{28}$

\section{A segurança alimentar e nutricional da população infanto-juvenil}

A SAN impõe compreensão abrangente do conceito de uma dieta adequada. Ela deve suprir as necessidades energéticas do indivíduo, ser nutricionalmente diversificada, respeitar a idade, as condições fisiológicas, a atividade física e, por fim, os hábitos culturais de cada um deles. ${ }^{6}$

No caso brasileiro, não há dúvidas de que a grande causa da falta de acesso aos alimentos, bem como da desnutrição infantil, é o baixo nível de renda. $\mathrm{O}$ acesso regular e adequado aos alimentos para as famílias dos estratos inferiores é custoso no sentido de que absorve parcela significativa da renda familiar e pode comprometer o acesso a outros bens e serviços necessários a uma vida digna. ${ }^{34}$

Sabe-se que no primeiro estágio de acesso à alimentação, em termos de poder aquisitivo, a dieta é mais restrita a fontes nutricionais menos custosas, como cereais, açúcar e produtos básicos. A partir de então, alimentos mais complexos e industrializados passam a ser paulatinamente incorporados, como o leite e seus derivados e carnes de aves, fontes de proteína animal, substituindo parte do consumo daqueles bens mais básicos. Em sequência, são consumidas outras fontes de proteína animal, como as carnes suína e bovina, e incorporam-se produtos hortícolas e frutas. ${ }^{35}$

No Brasil, segundo a POF 2008-2009, o consumo alimentar de leite é proporcional à renda. A despesa mensal familiar média é de $33 \%$ da renda. Comparando-se a maior e menor faixa de rendimento, a participação de leite e derivados na mesa dos brasileiros é três vezes maior nas famílias de maior renda. ${ }^{36}$

Sugere-se que os indivíduos com maior escolaridade fazem melhores escolhas alimentares para seu consumo. Porém, no Brasil, o índice de analfabetismo ainda é elevado, quando considerado o índice de 11,4\% diante de apenas $1 \%$ nos Estados Unidos, Reino Unido e Alemanha. ${ }^{35}$ Este fato demonstra a falta de aptidão de uma parte da população para realizar escolhas alimentares adequadas.

No Brasil, existem duas experiências de políticas públicas que colaboram para a SAN, por meio do consumo de leite para a população infantil. A modalidade de Incentivo à Produção e 
Consumo de Leite (PAA Leite) foi criada para contribuir com o aumento do consumo de leite bovino e/ou de cabra pelas famílias que se encontram em situação de insegurança alimentar e, ainda, incentivar sua produção por agricultores familiares. As famílias beneficiadas devem estar no limite máximo de renda per capita de até meio salário mínimo e ter, entre os membros da família, crianças entre dois e sete anos de idade, nutrizes até seis meses após o parto, gestantes e idosos a partir de 60 anos de idade. O PAA Leite atua no território da Superintendência do Desenvolvimento do Nordeste (SUDENE), sendo beneficiados todos os estados da Região Nordeste e também o norte de Minas Gerais. ${ }^{37}$

A segunda experiência trata do Programa Leite das Crianças (PLC), que foi instituído no âmbito do Estado do Paraná, pela Lei Estadual no 16.475/2010, ${ }^{38}$ como um direito de crianças de seis a 36 meses de idade, mães gestantes e nutrizes, com renda per capita de até meio saláriomínimo regional. O PLC consiste na distribuição gratuita e diária de um litro de leite pasteurizado, enriquecido com ferro quelato e vitaminas A e D. O programa objetiva auxiliar na redução das deficiências nutricionais da população infantil paranaense e propicia o estímulo ao desenvolvimento e organização da cadeia leiteira, com ênfase na geração de renda, criação de empregos, incentivando a permanência da população no interior do estado, como também a socialização de informações e procedimentos à inclusão social das famílias. ${ }^{38}$

Desta forma, políticas públicas exemplificadas pelos programas acima contribuem significativamente para a SAN e a realização do DHAA, pois promovem o acesso e a disponibilidade de nutrientes que auxiliam no suprimento das necessidades nutricionais da população infantil. Entretanto, se faz necessário implantar mais programas como os supramencionados em todas as regiões do país, assegurando que todas as crianças e adolescentes brasileiros sejam supridos com quantidade e qualidade não apenas desse alimento, mas de todos os outros, independentemente do poder aquisitivo das famílias. Devem ser implantadas, principalmente, políticas públicas específicas para o público infantil entre 6 e 12 meses, a fim de evitar alterações no sistema gastrointestinal e imunológico, no crescimento e no desenvolvimento infantil, garantindo assim a SAN do público infanto-juvenil.

\section{Considerações finais}

Dietas isentas e/ou insuficientes em leite podem contribuir para a desnutrição, retardo do crescimento e, ainda, favorecer outras reações adversas. A nutrição inadequada na infância pode ter efeitos adversos duradouros na saúde, mesmo na idade adulta. Estes efeitos podem ser reduzidos com estratégias voltadas para a SAN, que trazem inúmeros benefícios à saúde global do indivíduo, agregando qualidade de vida. 


\section{Referências}

1. Barroso GS, Sichieri R, Salles-Costa R. Fatores associados ao déficit nutricional em crianças residentes em uma área de prevalência elevada de insegurança alimentar. Rev. Bras. Epidemiol. 2008; 11(3):484-94.

2. Best C, Neufingerl N, van Geel L, van den Briel T, Osendarp S. The nutritional status of school-aged children: why should we care? Food Nutr Bull. 2010; 31(3):400-17.

3. Brasil. Ministério da saúde. PNDS. Pesquisa nacional de demografia e saúde da Criança e da Mulher 2006. Brasília: Ministério da saúde; 2009. 302 f.

4. Instituto Brasileiro de Geografia e Estatística. Pesquisa de orçamentos familiares 2008-2009. Antropometria e estado nutricional de crianças, adolescentes e adultos no Brasil. Rio de Janeiro: IBGE; 2010.

5. Instituto Brasileiro de Geografia e Estatística. Pesquisa Nacional por Amostra de Domicílios (PNAD). Segurança Alimentar 2004/2009. Rio de Janeiro: IBGE; 2010.

6. Brasil. Lei No 11.346, de 15 setembro de 2006. Sistema Nacional de Segurança Alimentar e Nutricional - SISAN com vistas em assegurar o direito humano à alimentação adequada e dá outras providências. Diário Oficial da União 18 set. 2006.

7. Arsenio L, Caronna S, Cioni F, Dall'aglio E. Homo sapiens and milk: a valuable food in the past and in the future. Mediterr. J. Nut. Metab. 2010; 3(2):99-103.

8. Medeiros LCS, Speridião PGL, Sdepanian VL, Fagundes Neto U, Morais MB. Ingestão de nutrientes e estado nutricional de crianças em dieta isenta de leite de vaca e derivados. J Pediatr 2004; 80(5):363-370.

9. Unicef. A convenção sobre os direitos da criança [Internet] 1989. [acesso em 04 abr. 2012]. Disponível em: http://www.unicef.pt/docs/pdf_publicacoes/convencao_direitos_crianca2004.pdf

10. Institute of Medicine. Dietary reference intakes for energy, carbohydrate, fiber, fat, fatty acids, cholesterol, protein and amino acids. Washington, DC.: The National Academy Press; 2005. 1331p.

11. Institute of Medicine. Dietary reference intakes for calcium and vitamin D [Internet]. Washington, DC.: IOM; nov. 2010. [acesso em 10 fev. 2012]. Disponível em: http://www.iom.edu/ /media/Files/ Report $\% 20$ Files/2010/Dietary-Reference-Intakes-for-Calcium-and-Vitamin-D/Vitamin \%20D\%20 and $\% 20$ Calcium $\% 202010 \% 20$ Report $\% 20$ Brief.pdf

12. Silva SMCS, Mura JDP. Tratado de alimentação, nutrição e dietoterapia. $2^{a}$ ed. São Paulo: Roca; 2010.

13. Drewnowski A. The contribution of milk and milk products to micronutrient density and affordability of the U.S. diet. J. Am. Coll. Nutr. 2011; 30 (5 Suppl 1):422S-8S.

14. Vissers PA, Streppel MT, Feskens EJ, Groot LC. The contribution of dairy products to micronutrient intake in the Netherlands. J Am Coll Nutr. 2011 oct; 30(5 Suppl 1):415S-21S.

15. Mills S, Ross RP, Hill C, Fitzgerald GF, Stanton C. Milk Inteligence: mining Milk for bioactive substances associated with human health. Int. Dairy J. 2011; 21(6):377-401.

16. Nicklas TA, O'neil CE, Fulgoni VL. The role of dairy in meeting the recommendations for shortfall nutrients in the American diet. J. Am. Coll. Nutr. 2009; 28(Suppl 1):73S-81S. 
17. Hidvégi E, Arató A, Cserháti E, Horváth C, Szabó A, Szabó A. Slight decrease in bone mineralization in cow milk-sensitive children. J. Pediatr. Gastroenterol. Nutr. 2003; 36(1):44-9.

18. Whiting SJ, Vatanparast H, Baxter-Jones A, Faulkner RA, Mirwald R, Bailey DA. Factors that affect bone mineral accrual in the adolescent growth spurt. J Nutr. 2004; 134(3):696S-700S.

19. Grillenberger M, Neumann CG, Murphy SP, Bwibo NO, Weiss RE, Jiang L, et al. Intake of micronutrients high in animal-source foods is associated with better growth in rural Kenyan school children. Br. J. Nutr. 2006; 95(2):379-90.

20. Dror DK, Allen LH. The importance of Milk and other animal-source foods for children in lowincome countries. Food Nutr. Bull. 2011; 32(3):227-43.

21. Beck-Nielsen SS. Rickets in Denmark. Dan. Med. J. 2012; 59(2):B4384.

22. Melamed ML, Kumar J. Low levels of 25-hydroxyvitamin D in the pediatric populations: prevalence and clinical outcomes. Ped. Health 2010; 4(1):89-97.

23. Prentice A. Vitamin D deficiency: a global perspective. Nutrition Reviews 2008; 66:S153-S164.

24. Kodama M, Uenishi K. Bone and joint diseases in children. Adequate calcium intake and dietary habit especially breakfast in children and adolescents. Clin. Calcium 2010; 20(6):867-71.

25. Vitolo MR. Nutrição: da gestação ao envelhecimento. Rio de Janeiro: Rubio; 2008.

26. Korhonen H, Pihlanto A. Bioactive peptides: production and functionality. Int. Dairy J. 2006; 16:945-960.

27. Vilela RM. The influence of whey peptides and fenretinide on inflammation and apoptosis in immortalized wild type and mutant F508 CFTR human tracheal epithelial cells. [tese]: School of Dietetics and Human Nutrition, McGill University; 2006.

28. Brunken GS, Silva SM, França GVA, Escuder MM, Venâncio SI. Fatores associados à interrupção precoce do aleitamento materno exclusivo e à introdução tardia da alimentação complementar no centro-oeste brasileiro. J. Pediatr 2006; 82(6):445-451.

29. Cruz MCC, Almeida JAG, Engstrom EM. Práticas alimentares no primeiro ano de vida de filhos de adolescentes. Rev. Nutr. 2010; 23(2):201-210.

30. Garcia MT, Granado FS, Cardoso MA. Alimentação complementar e estado nutricional de crianças menores de dois anos atendidas no Programa Saúde da Família em Acrelândia, Acre, Amazônia Ocidental Brasileira. Cad. Saúde Pública 2011; 27(2):305-316.

31. Oliveira MAA, Osório MM. Consumo de leite de vaca e anemia ferropriva na infância. J Pediatr 2005; 81(5):361-7.

32. Christie L, Hine RJ, Parker JG, Burks W. Food allergies in children affect nutrient intake and growth. J. Am. Diet Assoc. 2002; 102(11):1648-51.

33. Carvalho Junior FF. Apresentação clínica da alergia ao leite de vaca com sintomatologia respiratória. Pneumol. 2000; 27(1):17-24. 
34. Belik W. Perspectivas para segurança alimentar e. nutricional no Brasil. Saúde Soc. 2003; 12(1):12-20.

35. Brasil Food Trends 2020. São Paulo: Fiesp, Ital; 2010. [acesso em 07 jan. 2012]. Disponível em: http:// www.brasilfoodtrends.com.br/Brasil_Food_Trends/index.html

36. Brasil. Conselho Nacional de Segurança Alimentar e nutricional. A segurança alimentar e nutricional e o direito humano à alimentação adequada no Brasil. Indicadores e monitoramento da constituição de 1988 aos dias atuais. Brasília: Consea; 2010. 36 f.

37. Brasil. Lei n 10.696 , de 2 jul. de 2003. Dispõe sobre a repactuação e o alongamento de dívidas oriundas de operações de crédito rural, e dá outras providências 2003 jul 2. Diário Oficial da União 3 jul. 2003.

38. Paraná. Lei no 16.475, de 22 abril de 2010. Programa Leite das Crianças. Diário Oficial do Estado 22 abr. 2010; (8205):3.

Recebido: 01/3/2014

Revisado: $25 / 4 / 2014$

Aprovado: 30/4/2014 
